\title{
Strates
}

STRATES Matériaux pour la recherche en sciences sociales

$12 \mid 2006$

Nouvelles tensions impériales et recompositions en Europe centrale, orientale, et CEI

\section{Régions et nationalités en Russie : aspects institutionnels et juridiques}

\section{Anne GAZIER}

\section{(2) OpenEdition}

\section{Journals}

Édition électronique

URL : http://journals.openedition.org/strates/1702

DOI : $10.4000 /$ strates. 1702

ISSN : $1777-5442$

Éditeur

Laboratoire Ladyss

Édition imprimée

Date de publication : 31 décembre 2006

ISSN : 0768-8067

\section{Référence électronique}

Anne GAZIER, «Régions et nationalités en Russie : aspects institutionnels et juridiques », Strates [En ligne], 12 | 2006, mis en ligne le 19 juillet 2007, consulté le 08 septembre 2020. URL : http:// journals.openedition.org/strates/1702 ; DOI : https://doi.org/10.4000/strates.1702

Ce document a été généré automatiquement le 8 septembre 2020.

Tous droits réservés 


\title{
Régions et nationalités en Russie : aspects institutionnels et juridiques
}

\author{
Anne GAZIER
}

1 La question des nationalités est de toute évidence un des problèmes les plus épineux auxquels est confronté le pouvoir en Russie. On précisera au préalable que, conformément aux résultats du recensement de 1989, les citoyens de la Russie appartiennent à 128 nationalités différentes. Certes, les Russes forment $81,5 \%$ de la population et sont donc nettement majoritaires mais le poids des autres groupes ethniques est important dans certaines parties du territoire (notamment au Caucase nord et en moyenne Volga) ${ }^{1}$. La politique suivie à l'égard des nationalités autres que russe est d'abord marquée par un héritage soviétique contrasté. Ainsi, plus de 30 groupes ethniques (représentant $65 \%$ de l'ensemble des non-Russes) ont, à compter des années vingt et trente, été dotés d'un territoire qualifié d'« autonome " (république, district ou région, parfois bi- ou pluri-ethnique), ce qui leur a permis de préserver, dans une certaine mesure, leur culture ${ }^{2}$. Dans le même temps, une vaste entreprise de soviétisation et de russification a porté atteinte à l'identité linguistique, culturelle et religieuse de nombreux peuples. Il ne faut pas oublier, également, que certains d'entre eux (tels les Allemands de la Volga) ont été déportés sous Staline.

2 L'éclatement de l'URSS s'est accompagné, en Russie, de la montée en puissance des principales entités constituées sur une base ethnique (les républiques), qui ont très rapidement obtenu un accroissement considérable de leur autonomie en matière politique, économique et financière. A ainsi été instauré ce que l'on a appelé un "fédéralisme asymétrique », les républiques (couvrant 28,6\% de la superficie de la Russie et comprenant $15,2 \%$ de sa population) se voyant reconnaître, notamment dans le traité fédéral du 31 mars 1992, des droits nettement plus étendus que les autres entités régionales. La Constitution de 1993, tout en proclamant l'égalité de toutes les composantes de la Fédération (les 89 « sujets de la Fédération »), n’a pas supprimé tous les privilèges des républiques. En outre, elle a rendu possible un renforcement de leurs pouvoirs en permettant la conclusion de traités bilatéraux de délimitation des compétences entre les autorités fédérales et celles des sujets de la Fédération. Boris 
Eltsine a largement utilisé cette faculté et a, en particulier, signé avec les chefs des exécutifs de plusieurs républiques des traités qui leur ont conféré d'importantes attributions, réalisant une sorte de " fédéralisme à la carte ${ }^{3}$ ». Parallèlement, la volonté d'empêcher les discriminations fondées sur la nationalité a conduit, en 1997, à la décision de supprimer la mention de l'appartenance nationale dans le passeport intérieur (suscitant, cette fois-ci, le mécontentement des républiques qui ont tout de même obtenu qu'un document rédigé dans leur(s) langue(s) d'État puisse y être inséré). Enfin, la politique menée sous Boris Eltsine vis-à-vis des nationalités a comporté un nouveau volet lorsque, à partir de 1996, ont été adoptées des lois permettant à certains groupes ethniques (comme, par exemple, les "petits» peuples autochtones), de bénéficier de formes non territoriales d'autonomie.

3 L'arrivée au pouvoir de Vladimir Poutine et sa volonté d'affermir l'autorité du centre ont-elles entraîné la fin de cette période plutôt faste pour les peuples non russes de Russie? La réponse semble devoir être nuancée. D'un côté, en effet, on assiste à une diminution des prérogatives des entités territoriales dites nationales et, en particulier, des républiques, le président se montrant hostile tant au "fédéralisme asymétrique " qu'au «fédéralisme à la carte». De l'autre, en revanche, les autorités favorisent le renforcement de certains droits des nationalités, notamment ceux qui ne sont pas liés à un territoire.

Le déclin relatif des républiques et des autres entités territoriales " nationales "

4 Les 89 sujets de la Fédération se répartissent en deux grandes catégories: les entités «administratives" (6 territoires [krai], 49 régions [oblast] et 2 villes d'importance fédérale: Moscou et Saint-Pétersbourg) et les entités «nationales» (21 républiques, 1 région autonome et 10 districts [okroug] autonomes). Si l'on met à part la région autonome (c'est-à-dire la région autonome juive, les quatre autres régions autonomes que comptait la RSFSR ayant obtenu, en juillet 1991, leur transformation en républiques), les entités « nationales » comprennent, à leur tour, deux grands groupes : les républiques et les districts autonomes ${ }^{4}$. Ces derniers ne bénéficient pas d'un statut privilégié, bien au contraire. La Constitution de 1993 établit, en effet, qu'ils peuvent faire partie d'une région ou d'un territoire. C'est ainsi qu'à l'exception de la Tchoukotka qui est parvenue, en juin 1992, à sortir de la région de Magadan et à entrer directement dans la Fédération de Russie, les districts autonomes, pourtant reconnus sujets de la Fédération à part entière, sont inclus dans un autre sujet de la Fédération et donc placés sous sa juridiction. Il s'agit là d'une des contradictions majeures de la Constitution. Or, la tendance actuelle est à renforcer cette inégalité. Ainsi, un article d'une loi du 4 juillet 2003 prévoit que des compétences non négligeables seront exercées sur le territoire du district autonome par les organes dirigeants de sa région de rattachement, sauf si une loi fédérale en dispose autrement ${ }^{5}$. Par ailleurs, l'idée d'une fusion des sujets de la Fédération concernés fait son chemin : par exemple, le district autonome des Komi-Permiaks et la région de Perm ont lancé un processus tendant à transformer le district en simple division administrative de la région ${ }^{6}$. Cependant, cette évolution est loin de faire l'unanimité parmi les districts autonomes. Elle suscite même une franche hostilité chez ceux qui sont riches en matières premières et demeurent très attachés à leur autonomie.

5 La situation des républiques est bien différente. Tout d'abord, la Constitution de 1993 leur reconnaît, comme on l'a dit, des privilèges: qualifiées d'États, elles ont une Constitution (alors que les autres entités régionales adoptent un simple Statut); elles 
seules peuvent avoir une (ou plusieurs) langue(s) d'État en plus du russe. Ensuite, elles ont, pendant les années 1990, bénéficié d'un traitement de faveur qui leur a permis notamment de choisir librement leurs institutions (et de se doter, le plus souvent, d'un président élu au suffrage universel direct), d'adopter une constitution leur attribuant des pouvoirs parfois très étendus et, pour certaines d'entre elles, de conclure avec le pouvoir central un traité bilatéral leur conférant de larges prérogatives. Durant cette période, de grandes libertés ont couramment été prises avec la Constitution et les lois fédérales. Le souci de rétablir l'autorité du centre dans l'ensemble des régions de la Russie a conduit, en l'an 2000, au lancement, par V. Poutine, d'une vaste campagne visant à mettre en conformité la législation régionale avec le droit fédéral. À l'occasion de cette remise en ordre, les privilèges constitutionnels reconnus aux républiques ont fait l'objet d'une interprétation restrictive. Plus largement, la politique de recentralisation menée par le pouvoir a amené à traiter de la même façon tous les sujets de la Fédération et donc à supprimer, pour une bonne part, les particularités du statut des républiques.

Des privilèges constitutionnels interprétés restrictivement

6 À la différence du Traité fédéral du 31 mars 1992 qui consacrait la « souveraineté » des républiques et leur donnait des droits particulièrement étendus comme la propriété « de la terre, du sous-sol, des eaux et du monde végétal et animal» situés sur leur territoire ${ }^{7}$, la Constitution de 1993 ne leur attribue que des privilèges limités. En outre, la portée de ceux-ci tend à être réduite aussi bien par la Cour constitutionnelle de la Fédération de Russie que par la législation fédérale.

7 Tout d'abord, l'article $5 \$ 2$ de la Constitution, qui énumère les catégories de sujets de la Fédération, qualifie les seules républiques d'États. Quels droits ouvre ce statut étatique? Les républiques détiennent-elles une part de souveraineté ? Saisie de la question de la conformité de plusieurs constitutions de républiques à la Constitution de la Fédération de Russie, la Cour constitutionnelle de la Fédération de Russie a, dans deux décisions importantes rendues en juin $2000^{8}$, affirmé que les républiques ne peuvent se proclamer ni souveraines, ni même partiellement souveraines. Selon la Cour, en effet, seule la Fédération de Russie est souveraine. Il en résulte, en particulier, que les républiques ne peuvent se considérer comme des sujets de droit international ou comme propriétaires des ressources naturelles se trouvant sur leur territoire. De même, elles ne sauraient mettre en cause la primauté de la Constitution et des lois fédérales. Sont censurées également les dispositions des constitutions des républiques selon lesquelles le statut de celles-ci a un caractère contractuel. Se fondant sur cette interprétation, la Prokouratoura ${ }^{9}$ a exigé des républiques la révision de leur constitution ainsi que l'abrogation de la déclaration de souveraineté que nombre d'entre elles avaient adoptée au début des années 1990. Si la plupart des républiques se sont pliées à ces exigences ${ }^{10}$, certaines, comme le Tatarstan, ont adopté une nouvelle rédaction de leur Loi fondamentale réaffirmant leur souveraineté ${ }^{11}$. On observera, à cet égard, que les autorités fédérales ne donnent pas toujours l'exemple, la nouvelle Constitution de la Tchétchénie, rédigée sous leur contrôle et approuvée lors du référendum organisé le 23 mars 2003 dans la république, reconnaissant à celle-ci une souveraineté (certes très limitée...) ${ }^{12}$.

8 On peut rattacher également à cette conception stricte du caractère étatique des républiques la censure, par la Cour constitutionnelle, des dispositions de certaines de leurs constitutions qui soumettent à une condition de résidence au sein de la 
république la candidature aux élections à ses organes dirigeants (législatif ou exécutif) ${ }^{13}$. La Cour, qui invoque le principe d'égalité régissant le droit des citoyens de la Fédération de Russie d'être élus au sein des organes du pouvoir d'État, pose ici une sérieuse limite aux prérogatives des républiques à l'égard de leur territoire et de leur population.

9 Ensuite, l'article $68 \$ 2$ de la Constitution dispose que «les républiques ont le droit d'établir leurs langues d'État. Dans les organes du pouvoir d'État et les organes de l'auto-administration locale, les établissements d'État des républiques, elles sont utilisées parallèlement à la langue d'État de la Fédération de Russie». Plusieurs questions se posent à la lecture de ces dispositions :

10 - les républiques peuvent-elles librement se doter d'une (ou plusieurs) langue(s) d'État en plus du russe et réglementer leurs caractéristiques essentielles? La réponse qui découle des normes constitutionnelles paraît devoir être positive. Toutefois, une restriction a été introduite récemment en réaction contre l'adoption par le Tatarstan, en septembre 1999, d'une loi prévoyant l'abandon progressif du cyrillique par la langue tatare, au profit de la graphie latine. Ainsi, une loi du $1^{\text {er }}$ décembre 2002 qui a modifié la loi «sur les langues des peuples de la RSFSR » du 25 octobre 1991, impose désormais l'emploi du cyrillique par la langue d'État de la Fédération de Russie et les langues d'État des républiques, l'utilisation d'un autre alphabet ne pouvant être décidée que par une loi fédérale ${ }^{14}$;

11 - les républiques qui ont établi une ou des langues d'État peuvent-elles exiger des candidats à la fonction de chef de l'exécutif de la république la maitrise de cette (ou de ces) langue(s) en plus de celle du russe ? La Cour constitutionnelle de la Fédération de Russie n'a pas exclu une telle possibilité. Cependant, elle a considéré que seule une loi fédérale peut autoriser les républiques à poser ce type d'exigences et qu'une période transitoire doit être prévue pour que des programmes et des mesures permettent l'apprentissage et l'utilisation de la langue d'État de la république concernée. En l'absence d'une telle loi, elle a estimé contraire à la Constitution de la Fédération de Russie une disposition de la Constitution de la république des Adygués prévoyant la nécessité, pour pouvoir se présenter au poste de président de la république, de parler couramment les deux langues d'État de la république (l'adyguéen et le russe) ${ }^{15}$.

12 Enfin, et c'est là leur dernier privilège constitutionnel, les républiques adoptent une constitution et non un simple statut. Toutefois, la Constitution de la Fédération de Russie met sur un pied d'égalité ces deux types d'actes qui occupent, en particulier, la même place dans la hiérarchie des normes. Si, pendant longtemps, les républiques ont bénéficié d'une grande liberté pour choisir leurs dispositions constitutionnelles alors que les autres entités régionales se sont vues accorder une autonomie réduite dans l'adoption de leurs statuts, cette différence de traitement n'était nullement imposée par la Constitution. Elle a ainsi pu être mise en cause, à compter de la fin des années 1990, sans qu'il soit nécessaire de modifier la Loi fondamentale, lorsque le pouvoir central a cherché à reprendre en main l'ensemble des sujets de la Fédération.

Un statut de moins en moins particulier

Déjà, durant le second mandat de Boris Eltsine, des mesures ont atténué le statut privilégié des républiques. Ainsi, le procédé de l'élection s'est appliqué à l'ensemble des chefs des exécutifs régionaux (et non plus seulement à ceux des républiques). En outre, le Président de la Russie, qui avait nommé des représentants du président dans les territoires et les régions à la suite du putsch manqué d'août 1991, a entrepris d'en 
désigner également, à partir de 1996, dans les républiques. Surtout, les deux grandes lois adoptées en 1999 sur les compétences et sur les institutions des sujets de la Fédération ${ }^{16}$ ne font aucune différence entre les républiques et les autres entités régionales ${ }^{17}$. Avec l'arrivée au pouvoir de V. Poutine, cette tendance sera renforcée. En effet, la réforme institutionnelle lancée en l'an $2000^{18}$ non seulement concerne tous les sujets de la Fédération mais encore tend à une recentralisation et affecte donc tout spécialement les droits des entités les plus autonomes : les républiques. Notamment, depuis mai 2000, le pays est divisé en sept grands districts fédéraux avec à leur tête des représentants du président chargés, entre autres, d'assurer le respect des normes fédérales par les sujets de la Fédération compris dans leur circonscription. Les républiques sont particulièrement touchées par ce contrôle car ce sont elles qui ont adopté la législation la moins conforme à la Constitution et aux lois fédérales. De même, le droit dont dispose, à certaines conditions, le président de la Russie, depuis une loi en date du 29 juillet 2000, de destituer les chefs des exécutifs des sujets de la Fédération et de demander à la Douma la dissolution de leurs organes législatifs, constitue une menace destinée principalement à freiner les initiatives autonomistes des entités régionales et, notamment, des républiques. Plus récemment, une loi du 4 août 2001, qui centralise la direction de la police, diminue sensiblement les droits des républiques : ces dernières n'ont plus à donner leur accord pour la nomination de leurs ministres de l'Intérieur, qui incombe désormais au seul président de la Fédération de Russie (tout comme celle des chefs des directions de l'Intérieur des autres sujets de la Fédération) ${ }^{19}$.

La mise en cause des avantages accordés à des entités régionales va conduire par ailleurs le pouvoir central à s'engager, à compter de décembre 2001, dans un processus visant à l'abrogation des traités de délimitation des compétences conclus, de 1994 à 1998, entre la Fédération et une quarantaine de ses sujets ${ }^{20}$. Ce processus n'a pas laissé de côté les traités passés avec les républiques. Ainsi, 33 traités ont été abrogés, dont 5 conclus avec des républiques ${ }^{21}$. Toutefois, V. Poutine s'est contenté de signer, le 26 septembre 2002, avec les autorités de la république de Sakha (Iakoutie) un traité amendant le traité conclu antérieurement. Certes, les modifications apportées sont loin d'être négligeables : tant les compétences propres de la république que les compétences qu'elle partage avec la Fédération de Russie ont été nettement diminuées, en particulier dans les domaines fiscal, judiciaire et des relations internationales. Le traité ne déroge donc plus au droit fédéral mais il continue néanmoins à prendre en compte certaines spécificités de la république (comme sa richesse en métaux et en pierres précieuses ${ }^{22}$. En outre, quatre traités signés avec des républiques restent en vigueur et n'ont pas été, pour le moment au moins, modifiés ${ }^{23}$. Parmi ceux-ci figure le traité le moins conforme à la Constitution de la Fédération de Russie, celui conclu avec le Tatarstan, le 15 février 1994. On rappellera qu'il donne au Tatarstan des pouvoirs non prévus par la Constitution fédérale comme celui d'établir une citoyenneté "républicaine» ou d'avoir une banque nationale, et lui attribue en propre des compétences détenues par la Fédération (en matière de relations internationales, par exemple) ou des compétences conjointes (comme l'adoption des règles relatives à la possession, la jouissance et la disposition de la terre, du sous-sol, des eaux, des forêts et des autres ressources naturelles). Or le pouvoir central n'entend plus tolérer de telles violations des normes fédérales : ces traités sont appelés à être sinon abrogés, tout au moins renégociés et amendés à l'instar de celui conclu avec la république de Sakha (Iakoutie). 

tendance à la réduction de leurs droits. Il n'est pas question, cependant, pour le moment au moins, d'une remise en cause du découpage territorial de la Russie; la création de vastes provinces pluriethniques n'est pas à l'ordre du jour. Si l'on met à part le cas particulier des districts autonomes, l'existence même d'entités territoriales «nationales» ne paraît pas menacée, mais la préférence semble désormais aller au développement de formes non territoriales d'autonomie nationale, qui ne présentent pas de danger pour l'intégrité du pays.

L'accroissement des droits des nationalités non liés à un territoire présente comme première caractéristique d'ignorer la notion de minorité nationale. Pourtant, d'une part, la Constitution de 1993 prévoit, à son article 72, que «la réglementation et la protection des droits des minorités nationales " relèvent de la compétence de la Fédération; d'autre part, la Russie a ratifié, en 1998, la Conventioncadre du Conseil de l'Europe sur la protection des minorités nationales. Or l'absence de toute loi fédérale sur les minorités n'est compensée que partiellement par les textes qui, à compter de 1996, ont accru les droits de deux catégories de groupes ethniques ${ }^{24}$ : ceux qui sont appelés à bénéficier de «l'autonomie culturelle nationale » et ceux que l'on dénomme les "peuples autochtones peu nombreux », qui vivent principalement dans le Nord, en Sibérie et en Extrême-Orient.

Le développement de « l'autonomie cuturelle nationale »

17

La loi du 17 juin 1996 «Sur l'autonomie culturelle nationale » permet, selon son article premier, aux citoyens de la Fédération de Russie estimant appartenir «à des groupes ethniques déterminés " de s'associer afin de résoudre de façon autonome les questions liées «à la préservation de leur identité, au développement de leur langue, de l'éducation et de la culture nationale ${ }^{25} »$. Ils peuvent constituer des organisations (appelées " autonomies culturelles nationales») aux niveaux local, régional et fédéral. Les représentants des « autonomies » fédérales font partie d'un Conseil consultatif pour les autonomies culturelles nationales placé auprès du gouvernement de la Fédération de Russie, chargé notamment de participer à l'élaboration de programmes fédéraux et de projets d'actes normatifs concernant la langue et la culture des groupes ethniques.

Parmi les nombreux droits qui sont reconnus à ces organisations, on peut citer des droits assez généraux comme celui de s'adresser aux organes de l'État et de l'autoadministration locale pour y défendre les intérêts nationaux et culturels, de conserver et d'enrichir le patrimoine historique et culturel, d'observer les traditions et coutumes nationales. Mais on peut aussi relever des droits plus concrets comme celui de créer des médias, de recevoir et de diffuser les informations dans la langue nationale, de créer et de faire fonctionner des établissements d'enseignement ou à caractère scientifique ou culturel, de participer à l'action d'ONG en y désignant des représentants... Surtout, pour le financement de leurs programmes et de leurs projets, ces « autonomies culturelles nationales » ont droit à une aide de l'État (accordée par la Fédération ou par les sujets de la Fédération). C'est ce dernier aspect qui rend la formule attractive. Ainsi, ont été enregistrées plus de 300 organisations régionales et locales et 14 organisations fédérales. Au total, plus de 30 nationalités ont utilisé leur droit de constituer des " autonomies culturelles nationales ». Les groupes ethniques les plus actifs ont été les Allemands de la Russie et les Tatars, qui ont créé respectivement 68 et 63 organisations ${ }^{26}$. Toutefois, la loi de 1996 suscite d'importantes interrogations. 
Toutes les ethnies peuvent-elles, en effet, bénéficier de ses dispositions? Notamment les Russes ont-ils le droit de former des «autonomies culturelles nationales »? Ils ont utilisé cette faculté, en tout cas, dans des régions où ils sont pourtant majoritaires (comme les régions de Kourgan, de Vladimir, de Sverdlovsk, de Kaliningrad, etc.) ${ }^{27}$. Cependant, le ministère de la Justice de la Russie a refusé d'enregistrer l'organisation fédérale qu'ils ont constituée, en estimant que la loi de 1996 ne concerne pas le peuple russe qui représente la majeure partie de la population ${ }^{28}$. De même, au sein des républiques, les nationalités éponymes (dites «titulaires»), dont la langue et la culture font déjà l'objet d'une protection particulière, peuvent-elles créer des "autonomies culturelles nationales»? La loi de 1996 ne donne pas de réponse à cette question. Contrairement aux souhaits de certains ${ }^{29}$, elle ne limite pas son champ d'application aux seules ethnies qui se trouvent, dans un territoire donné, dans la situation d'une minorité nationale. On constate, malgré tout, que la plupart des groupes ethniques qui font usage des possibilités qu'elle offre sont des minorités. Parmi ces groupes figurent principalement des ethnies que l'on peut qualifier d'« exogènes » (c'est-à-dire dont le foyer principal se situe hors de Russie). Par exemple, les citoyens de Russie ukrainiens, kurdes, allemands, azerbaïdjanais, biélorusses, coréens et serbes ont formé des « autonomies culturelles nationales fédérales ${ }^{30}$ ». Cette loi n'est que rarement utilisée, en revanche, par les «petits peuples » indigènes du Nord et de Sibérie, dont les droits sont, il est vrai, protégés par une législation spécifique.

La reconnaissance de droits aux " petits » peuples

19 La Constitution de 1993 prévoit, à son article 69, que la Fédération de Russie " garantit les droits des peuples autochtones peu nombreux " mais il faudra attendre plusieurs années pour que le législateur fédéral précise ces dispositions. Pourtant, en 1995, la Douma a poussé un cri d'alarme. Elle a estimé que "la situation économique et culturelle des peuples autochtones (aborigènes) peu nombreux du Nord, de Sibérie et d'Extrême-Orient de la Fédération de Russie, qui comptent près de 200000 personnes, continue à se détériorer ». Elle a relevé, notamment, l'effondrement des branches d'activités économiques traditionnelles, l'augmentation du chômage et de la misère, l'aggravation de la situation sanitaire, le niveau de mortalité particulièrement élevé, les menaces pesant sur la survie de certains peuples. Et elle a préconisé toute une série de mesures urgentes, dont l'élaboration de plusieurs projets de loi ${ }^{31}$. Ces vœux seront partiellement exaucés à compter de la fin des années 1990, avec l'adoption de la loi du 30 avril 1999 « Sur les garanties des droits des peuples autochtones peu nombreux de la Fédération de Russie ${ }^{32}$ ", puis de deux lois venant préciser ou prolonger ses dispositions (mais uniquement pour les peuples du Nord, de Sibérie et d'Extrême-Orient), l'une relative aux communautés que peuvent constituer ces peuples, l'autre portant sur les territoires dont les ressources naturelles sont destinées à être exploitées par eux. Ces textes reconnaissent aux "petits peuples» des droits non négligeables mais qui comportent des limites importantes, notamment en matière administrative et politique.

20 L'essentiel des droits des petits peuples est fixé dans la loi du 30 avril 1999. On observera, au préalable, que le champ d'application de cette loi soulève moins de questions que celui de la loi de 1996 sur l'autonomie culturelle nationale. Son article premier précise, en effet, ce qu'il faut entendre par "peuples autochtones peu nombreux ». Il s'agit de peuples qui vivent dans les territoires où étaient établis leurs ancêtres, qui ont conservé un mode de vie et des activités traditionnels, qui comptent dans la Fédération de Russie moins de 50000 ressortissants et qui se considèrent 
comme des groupes ethniques. En outre, une liste unique de ces peuples est approuvée par le gouvernement de la Fédération de Russie. Celui-ci a ainsi adopté, le 24 mars 2000, une liste de 45 peuples $^{33}$. Toutefois, en raison de la présence au Daghestan d'une multitude d'ethnies différentes, il revient aux organes dirigeants de la république de fixer les caractéristiques - quantitatives et autres - de ses "peuples autochtones peu nombreux » et d'établir une liste spéciale (qui doit ensuite être incluse dans la liste unique). Le Conseil d'État du Daghestan s'est acquitté de cette tâche, mais dans la liste qu'il a adoptée le 18 octobre 2000, figurent... les Russes ${ }^{34}$ !

21 Le but de la loi du 30 avril 1999 est, conformément à son préambule, de garantir le développement socio-économique et culturel des peuples autochtones, de protéger leur habitat, leur mode de vie et leurs activités traditionnels. À ce titre leur sont reconnus non seulement des droits se rapportant à la culture (comme le droit de conserver et développer les langues maternelles, de créer des centres culturels, des médias, etc.) mais aussi des droits sociaux (comme celui de recevoir une assistance médicale gratuite) ou encore économiques. Notamment, ces peuples ont le droit de posséder et d'utiliser gratuitement, dans les lieux où ils sont établis de longue date, les terres indispensables à l'exercice de leurs activités traditionnelles ainsi que les ressources du sous-sol d'extraction courante. On doit mentionner également un droit plus spécifique reconnu aux personnes appartenant à ces peuples : le droit de faire un service civil à la place du service militaire.

Afin de préserver les traditions des peuples autochtones peu nombreux, la loi de 1999 prévoit, par ailleurs, que leurs ressortissants peuvent former des "communautés » (obchtchiny) sur la base soit de lignages, soit de liens de voisinage. Le statut de ces " communautés " a été précisé, pour les peuples du Nord, de Sibérie et d'ExtrêmeOrient, par une loi du 20 juillet $2000^{35}$. Celle-ci les définit comme des formes d'autoorganisation des personnes appartenant aux petits peuples, mais elle leur donne un rôle limité et spécialisé : les "communautés» ont principalement pour fonction d'organiser les activités économiques traditionnelles de leurs membres.

En outre, pour permettre à ces communautés et, plus généralement, aux personnes faisant partie des petits peuples d'exercer les droits que leur reconnait la loi de 1999 sur les terres de leurs ancêtres, une loi du 7 mai $2001^{36}$, qui limite à nouveau son champ d'application aux peuples du Nord, de Sibérie et d'Extrême-Orient, prévoit la création de sortes de réserves. Il s'agit de territoires dont les ressources naturelles sont mises gratuitement à la disposition des peuples autochtones afin qu'ils les exploitent de façon traditionnelle. Tant la formation de ces territoires que la détermination de leur régime juridique relèvent de la compétence des organes du pouvoir ${ }^{37}$, qui doivent toutefois recueillir au préalable l'avis de ressortissants des peuples autochtones et de leurs communautés. Les petits peuples sont ainsi associés - certes timidement - à la prise de décision dans un domaine les concernant au premier chef.

D'une façon générale, les droits politiques reconnus aux petits peuples comportent d'importantes limites. Tout d'abord, les lois fédérales ne leur reconnaissent pas le droit de former des organes locaux du pouvoir. En particulier, les communautés qu'ils peuvent constituer n'ont pas le statut de formations municipales et n'exercent donc pas l'auto-administration locale. Les organes de l'auto-administration locale peuvent tout au plus leur transférer certains de leurs pouvoirs. Certes, certains sujets de la Fédération (les républiques de Carélie, de Bouriatie et de Sakha, Iakoutie) ont créé des formations municipales «nationales-territoriales » là où vivent les petits peuples mais 
ces initiatives restent isolées ${ }^{38}$. Ensuite, les lois fédérales ne donnent aux «peuples autochtones peu nombreux » aucun droit à bénéficier d'une représentation particulière au sein des organes de l'État ou de l'auto-administration locale. Elles prévoient seulement la faculté, pour les sujets de la Fédération, de favoriser la représentation de ces peuples au sein de leurs organes représentatifs et de ceux de l'auto-administration locale grâce à l'établissement de quotas ou à la formation de circonscriptions électorales spéciales. En outre, les sujets de la Fédération ont la possibilité de former auprès de leurs organes exécutifs des conseils représentant ces peuples. Ainsi, tout dépend du bon vouloir des sujets de la Fédération. Or la législation régionale est très diverse dans ce domaine ${ }^{39}$. Enfin, la représentation des petits peuples au sein des organes du pouvoir est freinée par l'impossibilité pour leurs associations de se transformer en partis politiques, une loi du 11 juillet 2001 prohibant la formation de partis fondés sur l'appartenance nationale ${ }^{40}$.

Il apparaît ainsi que les textes reconnaissant des droits non liés à un territoire à certains groupes ethniques sont loin de concerner l'ensemble des minorités nationales et ne garantissent que de façon limitée la défense de leurs intérêts. En tout état de cause, ils ne sauraient donner aux nationalités des avantages équivalents à ceux que leur assure l'autonomie territoriale. On observe, en effet, que malgré les atteintes portées à leurs prérogatives, les entités territoriales dites nationales et, notamment, les républiques restent les mieux à même de protéger les droits des peuples non russes qu'elles comprennent. Du sort qui leur sera réservé dépend donc, dans une large mesure, la préservation de l'identité des nombreuses ethnies que comporte la Russie.

Juillet 2003

\section{NOTES}

1. Voir J. Radvanyi, La nouvelle Russie, Paris, Armand Colin, coll. U, 2000, p. 80-82.

2. Voir ibid., p. 82.

3. Sur cette évolution, voir notamment A. Gazier, « La réforme du système régional et local russe : un pas en avant, deux pas en arrière ", in R. Berton-Hogge, M.-A. Crosnier, Russie : la décentralisation aux prises avec l'étatisme, ex-URSS, édition 1994, Paris, La Documentation Française, p. 27-38 ; « Fédéralisme et auto-administration locale en Russie : un cadre juridique flou et ambigu », in J.-R. Raviot, « Russie : qui gouverne les régions? » Problèmes politiques et sociaux, $\mathrm{n}^{\circ}$ 783, 18 avril 1997, p. 11-19.

4. Pour des données politiques, économiques et sociales sur chacune des républiques que comprend la Russie, voir M.-A. Crosnier, « Multiple Russie : profils socioéconomiques des 21 républiques de la Fédération, de la Carélie... à la Iakoutie ", Le Courrier des Pays de l'Est, n 393 , octobre 1994, p. 3-69; pour une présentation détaillée de chacun des sujets de la Fédération, voir R. Brunet, La Russie. Dictionnaire géographique, CNRS-Libergéo/La Documentation française, coll. « Dynamiques du territoire », 2001, $480 \mathrm{p}$.

5. Il s'agit de l'article 26-6 de la loi du 4 juillet 2003 « Sur l'amendement de la loi fédérale » " Sur les principes généraux d'organisation des organes législatifs 
(représentatifs) et exécutifs du pouvoir d'État des sujets de la Fédération de Russie », Rossiïskaïa gazeta, 8 juillet 2003, p. 13.

6. Voir notamment Rossiïskaïa gazeta, 19 février 2003, p. 2.

7. Voir le texte de ce Traité in Le droit constitutionnel fédéral de la Russie. Sources essentielles (en russe), Norma, M., 1996, p. 189 et sq.

8. Il s'agit de l'arrêt de la Cour constitutionnelle de la Fédération du Russie du 7 juin 2000 «Sur l'examen de la constitutionnalité de plusieurs dispositions de la Constitution de la république de l'Altaï » et de la loi fédérale « Sur les principes généraux d'organisation des organes législatifs (représentatifs) et exécutifs du pouvoir d'État des sujets de la Fédération de Russie » (Sobranie zakonodatel'stva Rossiïskoï Federatsii - SzRF),2000, $\mathrm{n}^{\circ} 25$, art. 2728) et de l'ordonnance de la Cour du 27 juin 2000 "Sur la requête d'un groupe de députés de la Douma d'État tendant à l'examen de la conformité à la Constitution de la Fédération de Russie de certaines dispositions des Constitutions de la république des Adygués, de la république du Bachkortostan, de la république d'Ingouchétie, de la république des Komis, de la république d'Ossétie du Nord-Alanie et de la république du Tatarstan » (SzRF, 2000, n 29, art. 3117).

9. On rappellera que la Prokouratoura, dont l'origine remonte à Pierre le Grand, exerce deux fonctions essentielles : une fonction de ministère public et une fonction dite de « surveillance générale ». À ce dernier titre, cette institution vérifie notamment le respect de la légalité par les différentes administrations.

10. On peut citer l'exemple de la Bouriatie qui a abrogé, en avril 2002, la déclaration de souveraineté qu'elle avait adoptée en octobre 1990. Voir Rossiïskaïa gazeta, 18 avril 2002, p. 4.

11. Une véritable bataille judiciaire oppose, à ce propos, la Prokouratoura aux autorités du Tatarstan. La Prokouratoura conteste, en effet, la constitutionnalité de nombreuses dispositions de la nouvelle rédaction de la Constitution de la république, adoptée en avril 2002. Voir notamment Rossiïskaïa gazeta, 20 mars 2003, p. 3.

12. Cette souveraineté ne doit s'exercer, en effet, qu'en dehors des compétences de la Fédération et des compétences conjointes de la Fédération et de la république. Voir notamment Rossiïskaïa gazeta, 6 mars 2003, p. 4.

13. Voir, à titre d'exemple, l'arrêt de la Cour constitutionnelle de la Fédération de Russie du 24 juin 1997 « Sur l'examen de la constitutionnalité des dispositions des articles 74 (première partie) et 90 de la Constitution de la république de Khakassie », Vestnik Konstitoutsionnogo Souda Rossiïskoï Federatsii (VKSRF), 1997, n 5, p. 11 et sq.

14. Voir SzRF, 2002, n 50, art. 4926.

15. Voir l'ordonnance de la Cour constitutionnelle de la Fédération de Russie en date du 13 novembre 2001 « Sur la requête du Conseil de la république du Conseil d'État -

Khassé de la république des Adygués tendant à l'examen de la conformité à la Constitution de la Fédération de Russie du point 1 de l'article 76 de la Constitution de la république des Adygués ", VKSRF, 2002, n², p. 42 et sq.

16. Il s'agit de la loi du 24 juin 1999 « Sur les principes et les modalités de la répartition des domaines de compétence et des attributions entre les organes du pouvoir d'État de la Fédération de Russie et les organes du pouvoir d'État des sujets de la Fédération » (SzRF, 1999, n 26, art. 3176) et de la loi du 6 octobre 1999 « Sur les principes généraux d'organisation des organes législatifs (représentatifs) et exécutifs du pouvoir d'État des sujets de la Fédération de Russie » (SzRF, 1999, n 42, art. 5005).

17. Il en est de même de la loi précitée du 4 juillet 2003 qui a redéfini les rapports entre la Fédération et ses sujets. 
18. Sur cette réforme, voir notamment A. Gazier, « La mise au pas des régions russes? La réforme institutionnelle de Vladimir Poutine ", Le Courrier des Pays de l'Est, $\mathrm{n}^{\circ} 1015$, mai 2001, p. 4-14 ; J.-R. Raviot, « Les rapports centre-régions en Russie. Rééquilibrage et continuité », Le Courrier des Pays de l'Est, $\mathrm{n}^{\circ}$ 1033, mars 2003, p. 4-15.

19. Voir Rossiïskaïa gazeta, 9 août 2001, p. 6. De 1991 à 1999, les ministres de l'Intérieur des républiques étaient nommés par l'organe représentatif de celles-ci avec l'accord du ministre de l'intérieur de la Russie. À compter d'une loi du 31 mars 1999, ces responsables ont, à l'instar des chefs des directions de l'Intérieur des autres sujets de la Fédération, été nommés par le ministre de l'Intérieur de la Fédération de Russie avec l'accord du sujet de la Fédération concerné. Désormais, c'est le président de la Russie qui nomme l'ensemble de ces dirigeants sur proposition du ministre de l'Intérieur de la Russie, lequel doit juste solliciter l'avis du chef de l'exécutif du sujet de la Fédération concerné.

20. Voir la liste de ces 42 traités, in M. Lesage, Les institutions de la Russie, Documents d'études. Droit constitutionnel et institutions politiques, $\mathrm{n}^{\circ} 1.02$, édition 1998, p. 47-48. 21. Il s'agit des traités conclus avec les républiques Kabardino-Balkare, d'Ossétie du Nord-Alanie, de Bouriatie, des Komis, de Mari El.

22. Voir SzRF, 2002, nº 40, p. 9711.

23. Il s'agit des traités conclus avec les républiques du Tatarstan, du Bachkortostan, d'Oudmourtie et de Tchouvachie.

24. On mettra de côté, dans cette étude, la législation sur les Cosaques car elle présente trop de spécificités.

25. Voir la loi du 17 juin 1996 « Sur l'autonomie culturelle nationale », $S z R F, 1996, \mathrm{n}^{\circ} 25$, art. 2965.

26. Ces chiffres ont été donnés par V. Zorin, ministre (sans portefeuille) chargé de la politique des nationalités. Voir le communiqué de «RIA Oreanda » en date du 24 mai 2002.

27. Voir T. Ia. Khabrieva, «L'autonomie culturelle nationale: problèmes actuels de réglementation juridique " (en russe), Journal Rossiïkogo prava, n² 2, 2002, p. 11.

28. Voir M. Kouznetsov, « Discrimination » (en russe), Nezavisimaïa gazeta, 8 février 2001, p. 8.

29. Voir notamment T. Ia. Khabrieva, loc. cit., p. 11-12. On observera, à cet égard, qu'un projet de loi, adopté en seconde lecture par la Douma le 27 septembre 2002, apporte des modifications et des précisions à la loi sur l'autonomie culturelle nationale mais ne restreint pas son champ d'application aux minorités.

30. Quant à la région de Moscou, elle comptait, dès 1998, 11 « autonomies culturelles nationales » qui étaient formées par les citoyens de Russie arméniens, assyriens, juifs, kazakhs, coréens, kurdes, lettons, allemands, polonais, serbes et bulgares. Voir M. Kouznetsov, loc.cit.

31. Voir l'arrêté de la Douma d'État de l'Assemblée fédérale de la Fédération de Russie du 26 mai 1995 «Sur la situation de crise de l'économie et de la culture des peuples autochtones (aborigènes) peu nombreux du Nord, de Sibérie et d'Extrême-Orient de la Fédération de Russie ", SzRF, 1995, n 24, art. 2260.

32. Voir SzRF, 1999, no 18, art. 2208.

33. Voir l'arrêté du gouvernement de la Fédération de Russie du 24 mai 2000, «Sur la liste unique des peuples autochtones peu nombreux de la Fédération de Russie ", SzRF, 2000, n 14 , art. 1493. 
34. Voir l'intervention de V. A. Kriajkov lors de la table ronde sur « Les standards du Conseil de l'Europe dans le domaine de la protection des minorités nationales et la pratique de la Russie ", organisée en 2001 par l'Institut du droit et de la politique publique, site www.tolerance.ngo.ru (accessible en mars 2006).

35. Voir la loi du 20 juillet 2000 «Sur les principes généraux d'organisation des communautés des peuples autochtones peu nombreux du Nord, de Sibérie et d'Extrême-Orient de la Fédération de Russie ", SzRF, 2000, n 30, art. 3122.

36. Voir la loi du 7 mai 2001 «Sur les territoires destinés à l'exploitation traditionnelle des ressources naturelles par les peuples autochtones peu nombreux du Nord, de Sibérie et d'Extrême-Orient de la Fédération de Russie ", SzRF, 2001, n 20, art. 1972. 37. Les territoires concernés pouvant être fédéraux, régionaux ou locaux, les organes du pouvoir compétents pour les créer et fixer leur régime juridique sont, suivant les cas, le gouvernement de la Fédération de Russie, les organes exécutifs des sujets de la Fédération ou les organes de l'auto-administration locale.

38. Voir V. A. Kriajkov, « La participation des peuples autochtones peu nombreux à la formation de la volonté politique - questions étatiques et juridiques » (en russe), Gosoudarstvo i pravo, $\mathrm{n}^{\circ} 1,2000, \mathrm{p} .21$.

39. Voir ibid., p. 21-22. En outre, l'application de cette législation soulève parfois des problèmes. Par exemple, l'un des sujets de la Fédération les plus actifs dans la défense des droits des petits peuples, le district autonome des Khanty-Mansi a créé une « Assemblée de représentants des peuples autochtones peu nombreux » qui est un organe consultatif placé auprès de la Douma du district. Cependant, le système électoral adopté pour former cette « Assemblée » a conduit à l'élection de seulement 4 députés issus des petits peuples sur un total de $25 .$. Voir Rossiïskaïa Federatsiïa Segodnia, $\mathrm{n}^{\circ}$ 5, 2002, p. 56-57.

40. Voir l'article 9 point 3 de la loi du 11 juillet 2001 «Sur les partis politiques », SzRF, $2001, n^{\circ} 29$, art. 2950.

\section{RÉSUMÉS}

Ce nouveau découpage établi par la Constitution de 1993, en cours de révision par le président Vladimir Poutine, est très clairement bien exposé dans sa logique refondatrice par Anne Gazier qui en montre l'évolution sous les deux présidences. Le système de catégorisation interethnique préconisé par le nouveau pouvoir redonne toute leur place aux petits groupes ethniques maltraités sous le communisme et dont les revendications sont basées sur des espoirs de réparation historique et sur les privilèges qui sont associés aux statuts attribués.

Anne Gazier.Relationships between State/nation/regions/ethnos in post-sovietique space

The author presents the new territorial division established by the constitution of 1993, now being revised by President Putin. She explains the founding ideology behind it as well as its evolution under the two presidencies. The system of inter-ethnic categorisation put forward by the new power gives their place to the small ethnic groups badly treated by communism, and whose claims are built on hopes of historical reparation and its associated privileges. 
AUTEUR

ANNE GAZIER

Maître de conférence de droit public, université de Paris X, anne.gazier@wanadoo.fr 